\title{
Primary thyroid paraganglioma mimicking medullary thyroid carcinoma: A case report
}

\author{
$\mathrm{XING} \mathrm{YU}^{1}$, YONG WANG ${ }^{1}$, PING WANG $^{1}$, CAI-HONG JI ${ }^{2}$, CHUN-DI MIAO $^{2}$ and SHU ZHENG $^{3}$ \\ ${ }^{1}$ Department of Surgery, The Second Affiliated Hospital, College of Medicine, Zhejiang University, Hangzhou, Zhejiang \\ 310009; ${ }^{2}$ College of Medicine, Zhejiang University, Hangzhou, Zhejiang 310058; ${ }^{3}$ Department of Oncology, \\ The Second Affiliated Hospital, College of Medicine, Zhejiang University, Hangzhou, Zhejiang 310009, P.R. China
}

Received September 20, 2014; Accepted April 30, 2015

DOI: $10.3892 / \mathrm{ol} .2015 .3292$

\begin{abstract}
Primary thyroid paraganglioma (TP) is an uncommon tumor, and in rare cases, this disease tends to mimic medullary thyroid carcinoma (MTC). The present study reports a rare case of primary TP mimicking thyroid carcinoma, accompanied by hyperthyroidism. A 30-year-old female presented with an anterior cervical mass. Pre-operative radiological studies and operative frozen section analysis indicated an atypical MTC. Primary TP was finally diagnosed by pathology and immunohistochemical staining. Laboratory examinations (thyroid hormones tests) and Tc99m emission computed tomography revealed hyperthyroidism. Gene analysis of TP-associated gene mutations was negative. Surgical resection was performed as a curative approach and there is currently no metastasis after 36 months of follow-up. Surgeons must be aware of this disease in order to ensure a correct diagnosis and to prevent them from performing unnecessary procedures. The current study presents a case of primary TP mimicking MTC, discusses the radiographic results and histological characteristics, and provides a review of the associated literature.
\end{abstract}

\section{Introduction}

Thyroid paraganglioma (TP) is an uncommon tumor, which may be derived from the inferior laryngeal paraganglia (1). Paraganglioma is a neuroendocrine tumor originating from paraganglia of the autonomic nervous system, which is derived from ectoderm cells (2), whereas the thyroid gland is derived from cells of entodermal origin (3) and thus, it would appear

Correspondence to: Dr Yong Wang, Department of Surgery, The Second Affiliated Hospital, College of Medicine, Zhejiang University, 88 Jiefang Street, Hangzhou, Zhejiang 310009, P.R. China

E-mail: surgwy@163.com

Key words: histological characteristics, thyroid paraganglioma, medullary thyroid carcinoma, hyperthyroidism status, radiographic results that the development of paraganglioma originating from the thyroid gland is extremely unlikely. Therefore, at present, the pathogenesis of TP remains unclear. However, in the English literature, $<40$ documented cases have been reported. As described in previous reports, TP is generally slow-growing and most patients present with an asymptomatic neck mass (1). On ultrasonography, TP typically appears as a solid hypoechoic nodule, showing no significant differences from other common types of thyroid neoplasms. Computed tomography (CT) generally displays a thyroid mass of low density, and thus TPs are usually mistaken as benign tumors (4). Surgery is generally the recommended treatment method, and few cases involving local recurrence or metastasis following surgical mass excision have been reported. In certain cases, TP can mimic medullary thyroid carcinoma (MTC) (4-8). MTC arises from parafollicular cells and exhibits similar microscopic features to TP, with a nesting or organoid pattern of growth under histological examination (7). However, the presentation of TP concomitant with hyperthyroidism has never been reported. The present study reports the first case of primary TP mimicking MTC accompanied by hyperthyroidism. This study was approved by the Ethics Committee of The Second Affiliated Hospital, College of Medicine, Zhejiang University (Hangzhou, China). Consent was obtained from the patient's family.

\section{Case report}

On July 16th, 2009, a 30-year-old female with an enlarging anterior cervical mass, a hoarse voice and weight loss that had been apparent for 2 months was admitted to the Department of Thyroid Surgery of the Second Affiliated Hospital, College of Medicine, Zhejiang University (Hangzhou, China). CT revealed a left thyroid nodule, which was a $2.8 \times 1.2 \times 1.7-\mathrm{cm}$ non-calcific solid mass with low density, and ultrasonography showed that the elastic grade was grade 2 (Fig. 1). Tc99m emission computed tomography (ECT) demonstrated hyperthyroidism and left thyroid 'cold nodules'. The laboratory examination results for serum free triiodothyronine (FT3), free thyroxine (FT4) and human-thyroid-stimulating hormone (h-TSH) levels were recorded as $12.80 \mathrm{pmol} / 1$ (normal range, 3.5-6.5 pmol/1), $35.44 \mathrm{pmol} / \mathrm{l}$ (normal range, 8.9-20.6 pmol/l) and $0.02 \mathrm{mIU} / \mathrm{l}$ (normal range, 0.35-4.6 mIU/1), respectively. Following the treatment of propylthiouracil (100 mg, every $8 \mathrm{~h}$ for 4 weeks) 

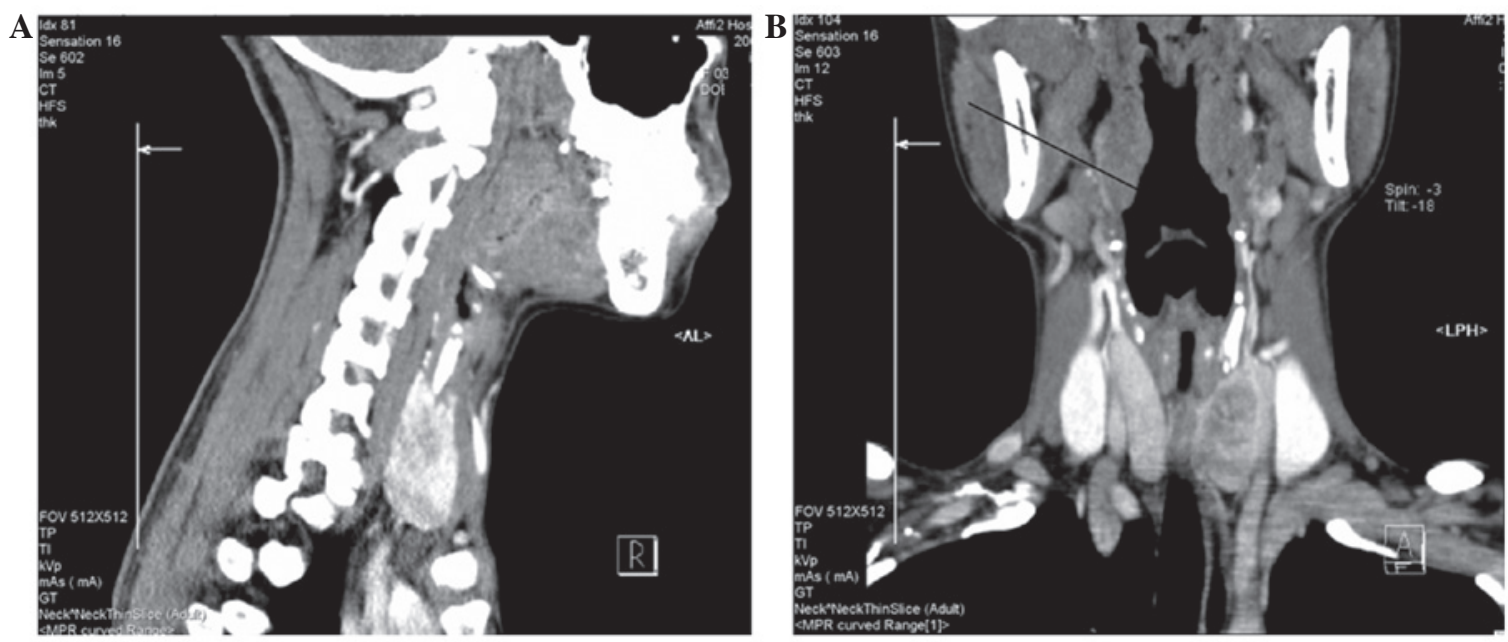

Figure 1. Computed tomography (CT) scans showing a 2.8x1.2x1.7-cm non-calcified solid nodule inside the left thyroid lobe without any extension. (A) Sagittal and (B) coronal planes, showing heterogenous density of the thyroid nodule on enhanced CT scan.
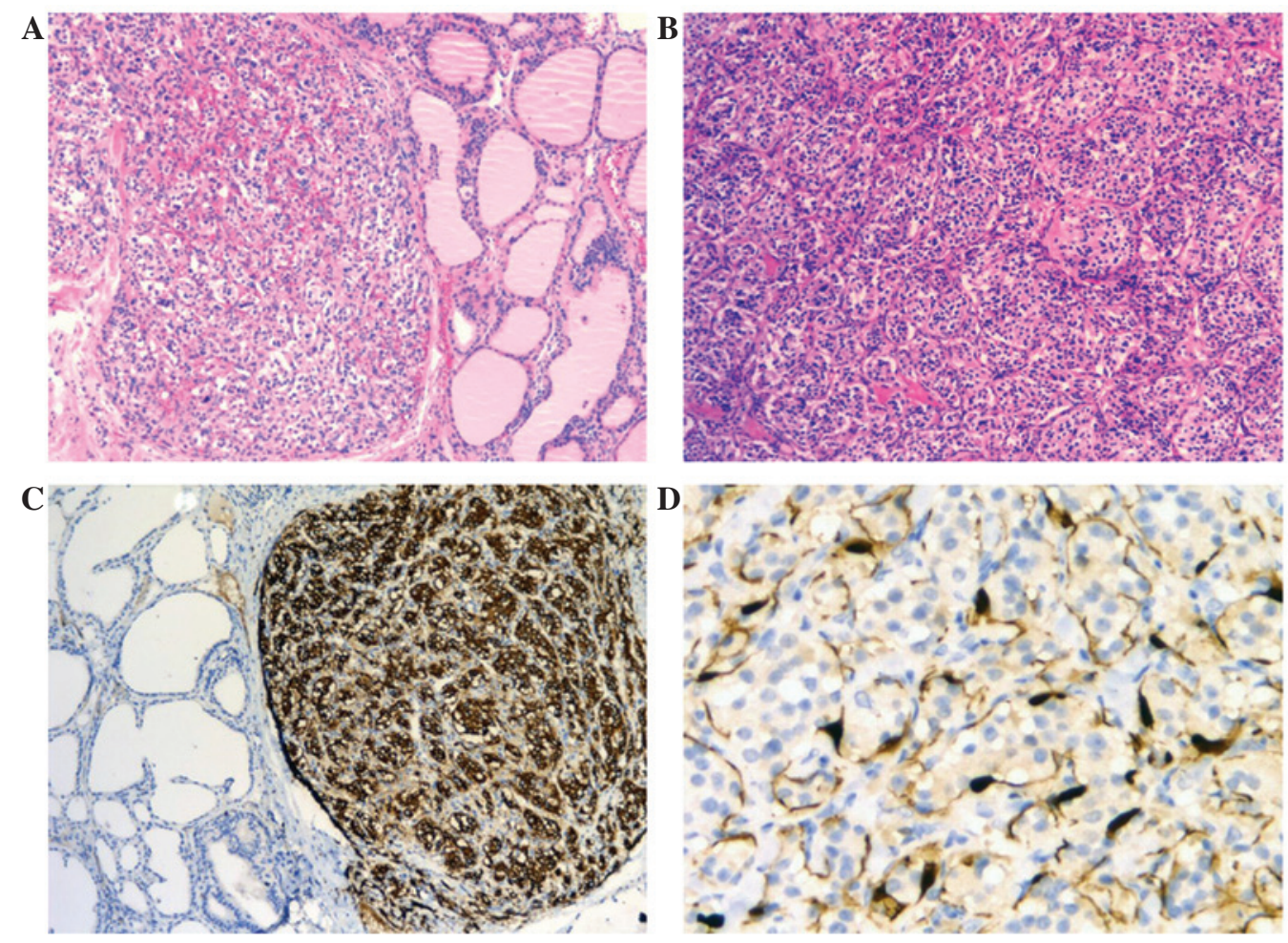

Figure 2. Histological findings of the thyroid mass after thyroidectomy. (A) Neoplasm showing nesting and a zellballen growth pattern in the normal thyroid follicles [hematoxylin and eosin (HE) stain; original magnification, x100). (B) Tumor cells with round nuclei and basophilic cytoplasm, with a characteristic nesting pattern (HE stain; original magnification, x200). (C) Chromogranin A-positive tumor cells [immunohistochemical (IHC) stain; original magnification, $\mathrm{x} 100]$. (D) Sustentacular cells highlighted by S-100 protein (IHC stain; original magnification, $\mathrm{x} 40$ ).

for hyperthyroidism, a pre-operative fine-needle aspiration cytology (FNAC) biopsy was performed, but was deemed to have no diagnostic value due to the presence of excessive blood.

Finally, the patient chose surgery rather than FNAC reexamination. The pre-operative measurements of the FT3, FT4 and h-TSH levels were $5.10 \mathrm{pmol} / 1,14.68 \mathrm{pmol} / \mathrm{l}$ and $0.02 \mathrm{mIU} / \mathrm{l}$. During the left hemithyroidectomy, the mass was observed to be involved in the left thyroid capsule. The mass was grossly described as a $2.5 \times 1.2 \times 1.5-\mathrm{cm}$ solitary gray-red nodule, with multiple areas of hemorrhage and necrosis.
Frozen section analysis during the procedure revealed solid nests of cells with slight pleomorphic nuclei. Tumor cells appeared to be malignant, with the pattern of necrosis and vascular invasion, initially suggesting a diagnosis of MTC. The histological characteristics of the presumptive MTC were not typical and, following communication with the patient's family, a left thyroidectomy and central lymph node dissection was performed.

The final histological examination showed distinctive cell nests (with zellballen architecture), but none of the 9 lymph 
nodes were involved. The majority of tumor cells were small and round with rich basophilic cytoplasm. Based on the typical zellballen growth pattern of the neoplasm, primary TP was determined in the final pathological diagnosis and was confirmed by positive immunohistochemical staining (IHC) for neuron-specific enolase, synaptophysin, chromogranin A and S-100, and negative staining for carcinoembryonic antigen and calcitonin (Fig. 2).

In the long-term post-operative surveillance, ultrasonography of the neck, CT of the chest and abdomen, and the 24-h urine catecholamine level were normal. The surgery also cured the hyperthyroidism, and the latest results of the serum FT3, FT4 and h-TSH tests were $4.15 \mathrm{pmol} / 1,14.54 \mathrm{pmol} / 1$ and $1.96 \mathrm{mIU} / 1$, respectively. The patient also received genetic counseling and the subsequent genetic test was negative for RET, SDHB and SDHD mutations.

The patient is currently doing well without any evidence of metastasis at the time of the last follow-up at 36 months post-surgery.

\section{Discussion}

Thyroid nodular lesions mimicking MTC are rare, and obtaining the correct diagnosis and making the appropriate surgical decisions is challenging for surgeons. To date, the pre-operative diagnosis of TP by clinical findings and radiological examinations remains difficult $(5,6,9)$. The morphology of TP is difficult to distinguish from that of MTC and thus, pre-operative FNAC biopsy or intraoperative frozen section analysis rarely report a correct diagnosis (4). However, IHC is of great use in differentiating TP from other thyroid neoplasms, such as hyalinizing trabecular adenoma and MTC (9).

The current study presents a case of primary TP that mimicked MTC. This is also the first case exhibiting TP accompanied by hyperthyroidism. According to the imaging manifestation the present case, in hyperthyroidism, the thyroid lobes have a rich vascular supply. The congestive status of the lobe indirectly enhances the blood flow signal of the thyroid nodule, which confuses the surgeons in assessing the properties of the nodules. Therefore, as in the present case, ultrasonography shows an abundant blood flow signal in the paraganglioma and the thyroid lobes. In the contrast-enhanced CT scans of the present study, the paraganglioma and the thyroid lobes exhibited enhancement, although the degree of enhancement in the paraganglioma was less than that in the thyroid lobes. ECT revealed hyperthyroidism and thyroid 'cold nodules'.

With regard to the gross anatomy, the paraganglioma was absolutely involved in the left thyroid lobe in the present case. The diagnosis of TP was further confirmed by pathology and IHC. As repeated examinations of the neck, chest and abdomen presented no other tumors, metastasis from another location was excluded.

Primary TP possesses unknown malignant potential, however, to date, no studies have reported locoregional recurrence or distant metastasis, despite records of a few tumors that displayed questionable morphological features (6). The literature provides good evidence that extensive surgical resection can achieve a long disease-free interval $(5,7,10,11)$. Although irradiation has been demonstrated to be effective for enlarging and symptomatic paragangliomas $(12,13)$, an indication for primary radiotherapy is established rarely, and only if a surgical procedure is likely to involve a high risk of damage to several vessels and nerves (14). Moreover, as the present patient was negative for RET, SDHB or SDHD mutations $(15,16)$, a left hemithyroidectomy without radiotherapy was appropriate.

In conclusion, the present study reports a rare case of primary TP accompanied by hyperthyroidism. This case aims to raise awareness of this entity, which can be mistaken for MTC. IHC plays a significant role in the definitive diagnosis, and gene analysis, such as RET and SDH mutation detection, is a factor to consider when adjusting the treatment plan and evaluating the prognosis.

\section{Acknowledgements}

This study was supported by the Natural Science Foundation of Zhejiang Province (grant no. LY14H160033).

\section{References}

1. Kieu V, Yuen A, Tassone P and Hobbs CG: Cervical paraganglioma presenting as thyroid neoplasia. Otolaryngol Head Neck Surg 146: 516-518, 2012.

2. Holt DE, Henthorn P, Howell VM, et al: Succinate dehydrogenase subunit D and succinate dehydrogenase subunit B mutation analysis in canine phaeochromocytoma and paraganglioma. $\mathrm{J}$ Comp Pathol 151: 25-34, 2014.

3. Besova NV, Savel'ev SV, Istomin AA and Chernikov VP: An immunohistochemical study of the primordium and primary differentiation of the parafollicular cells in the human thyroid. Izv Akad Nauk SSSR Biol: 615-619, 1990 (In Russian).

4. Yu BH, Sheng WQ and Wang J: Primary paraganglioma of thyroid gland: A clinicopathologic and immunohistochemical analysis of three cases with a review of the literature. Head Neck Pathol 7: 373-380, 2013.

5. Akhtar K, Sen Ray P, Ahmad SS and Sherwani RK: Paraganglioma of the thyroid gland: Cytologists' enigma. BMJ Case Rep 2013: bcr2013009518, 2013.

6. Kronz JD, Argani P, Udelsman R, et al: Paraganglioma of the thyroid: Two cases that clarify and expand the clinical spectrum. Head Neck 22: 621-625, 2000.

7. Ferri E, Manconi R, Armato E and Ianiello F: Primary paraganglioma of thyroid gland: A clinicopathologic and immunohistochemical study with review of the literature. Acta Otorhinolaryngol Ital 29: 97-102, 2009.

8. Jeong YJ, Oh HK and Bong JG: Multiple endocrine neoplasia type 1 associated with breast cancer: A case report and review of the literature. Oncol Lett 8: 230-234, 2014.

9. Phitayakorn R, Faquin W, Wei N, et al: Thyroid-associated paragangliomas. Thyroid 21: 725-733, 2011.

10. Corrado S, Montanini V, De Gaetani C, et al: Primary paraganglioma of the thyroid gland. J Endocrinol Invest 27 : 788-792, 2004.

11. Erem C, Kocak M, Nuhoglu I, et al: Primary thyroid paraganglioma presenting with double thyroid nodule: A case report. Endocrine 36: 368-371, 2009.

12. Krych AJ, Foote RL, Brown PD, et al: Long-term results of irradiation for paraganglioma. Int J Radiat Oncol Biol Phys 65: 1063-1066, 2006.

13. Kunzel J, de Tristan J, Mantsopoulos K, et al: Experiences in the treatment of patients with multiple head and neck paragangliomas. Am J Otolaryngol 35: 294-299, 2014.

14. Kunzel J, Koch M, Brase C, et al: Treatment of cervical paragangliomas: Is surgery the only way? Am J Otolaryngol 35: 186-191, 2014.

15. Zantour B, Guilhaume B, Tissier F, et al: A thyroid nodule revealing a paraganglioma in a patient with a new germline mutation in the succinate dehydrogenase B gene. Eur J Endocrinol 151: 433-438, 2004.

16. Moraitis AG, Martucci VL and Pacak K: Genetics, diagnosis, and management of medullary thyroid carcinoma and pheochromocytoma/paraganglioma. Endocr Pract 20: 176-187, 2014. 